\title{
Review Article \\ Fluctuation Analyses for Pattern Classification in Nondestructive Materials Inspection
}

\author{
A. P. Vieira, ${ }^{1}$ E. P. de Moura, ${ }^{2}$ and L. L. Gonçalves ${ }^{2}$ \\ ${ }^{1}$ Instituto de Física Universidade de São Paulo, 05508-090 São Paulo, SP, Brazil \\ ${ }^{2}$ Departamento de Engenharia Metalúrgica e de Materiais, Universidade Federal do Ceará, 60455-760 Fortaleza, CE, Brazil
}

Correspondence should be addressed to L. L. Gonçalves, lindberg@fisica.ufc.br

Received 30 December 2009; Accepted 25 June 2010

Academic Editor: João Marcos A. Rebello

Copyright (C) 2010 A. P. Vieira et al. This is an open access article distributed under the Creative Commons Attribution License, which permits unrestricted use, distribution, and reproduction in any medium, provided the original work is properly cited.

\begin{abstract}
We review recent work on the application of fluctuation analyses of time series for pattern classification in nondestructive materials inspection. These analyses are based on the evaluation of time-series fluctuations across time intervals of increasing size, and were originally introduced in the study of fractals. A number of examples indicate that this approach yields relevant features allowing the successful classification of patterns such as (i) microstructure signatures in cast irons, as probed by backscattered ultrasonic signals; (ii) welding defects in metals, as probed by TOFD ultrasonic signals; (iii) gear faults, based on vibration signals; (iv) weld-transfer modes, as probed by voltage and current time series; (v) microstructural composition in stainless steel, as probed by magnetic Barkhausen noise and magnetic flux signals.
\end{abstract}

\section{Introduction}

Many nondestructive materials-inspection tools provide information about material structure in the form of time series. This is true for ultrasonic probes, acoustic emission, magnetic Barkhausen noise, among others. Ideally, signatures of material structure are contained in any of those time series, and extracting that information is crucial for building a reliable automated classification system, which is as independent as possible from the operator's expertise.

As in any pattern classification task, finding a set of relevant features is a key step. Common in the literature are attempts to classify patterns from time series by directly feeding the time series into neural networks, by measuring statistical moments, or by employing Fourier or wavelet transforms. These last two approaches are hindered by the presence of noise, and by the nonstationary character of many time series. Sometimes, however, relevant information is hidden in the "noise" itself, as this can reflect memory effects characteristic of underlying physical processes. Analysis of the statistical properties of the time series can reveal such effects, although global calculations of statistical moments miss important local details. Here, we show that properly defined local fluctuation measures of time series can yield relevant features for pattern classification. Such fluctuation measures, which are sometimes referred to as "fractal analyses", were introduced in the study of mathematical fractals, objects having the property of scale invariance. It turns out that they can also be quite useful in the study of general time series. Early applications [1-3] of fluctuation analyses to defect or microstructure recognition relied on extracting exponents and scaling amplitudes expected to characterize memory effects on various systems. The approach reviewed here, on the other hand, is based on more general properties of the fluctuation measures.

The remaining of this paper is organized as follows. In Section 2, we define mathematically the fluctuation (or fractal) analyses used to extract relevant features from the various time series. In Section 3, we review the tools used in the proper pattern-classification step, illustrated by several applications in Section 4. We close the paper by presenting our conclusions in Section 5.

\section{Fluctuation Analyses}

All techniques of fluctuation analysis employed here start by dividing the signal into time intervals containing $\tau$ 
points. Each technique then involves the calculation of the average of some fluctuation measure $Q(\tau)$ over all intervals, for different values of $\tau$, thus gathering local information across different time scales. For a signal with genuine fractal features, $Q(\tau)$ should scale as a power of $\tau$,

$$
Q(\tau) \sim \tau^{\eta},
$$

at least in an intermediate range of values of $\tau$, corresponding to $1 \ll \tau \ll L, L$ being the signal length.

In general, the exponent $\eta$ is related to the so-called Hurst exponent $H$ of the time series [4, 5]. This exponent is expected to gauge memory effects which somehow reflect the underlying physical processes influencing the signal. A simple example is provided by fractional Brownian motion [5-7], in which correlated noise is postulated, leading to persistent or antipersistent memory, and to a standard deviation $\sigma(t)$ following:

$$
\sigma(t)=\left(2 K_{f} t\right)^{H},
$$

where $t$ is the time elapsed since the motion started, and $K_{f}$ is a generalized diffusion coefficient. A Hurst exponent equal to $1 / 2$ corresponds to regular Brownian motion, while values of $H$ different from $1 / 2$ indicate the presence of long-range memory mechanisms affecting the motion; $H>(1 / 2)(H<$ $1 / 2$ ) corresponds to persistent (antipersistent) behavior of the time series.

Real-world time series, however, originate from a much more complex interplay of processes, acting at different characteristic time scales, and which, therefore, compete to induce memory effects whose nature may change as a function of time. As the series is probed at time intervals of increasing size, the effective Hurst exponent can vary. In that case, any other exponent $\eta$ related to $H$ would likewise vary. This variation of $\eta$ with the size $\tau$ of the time interval is precisely what the present approach exploits.

Once the relevant features are obtained from the variation of $\eta$ with $\tau$, the different patterns can be classified with the help of statistical tools available in the patternrecognition literature. Here, as discussed in Section 3, we make use of principal component analysis (PCA) and Karhunen-Loéve transformations. (See, e.g., [8] for a thorough account of statistical pattern classification).

2.1. Hurst (R/S) Analysis. The rescaled-range (R/S) analysis was introduced by Hurst [4] as a tool for evaluating the persistency or antipersistency of a time series. The method works by calculating, inside each time interval, the average ratio of the range (the difference between the maximum and minimum values of the accumulated series) to the standard deviation. The size of each interval is then varied.

Mathematically, the R/S analysis is defined in the following way. Given an interval $I_{k}$ of size $\tau$, we calculate $\langle z\rangle_{\tau, k}$, the average of the series $z_{i}$ inside that interval,

$$
\langle z\rangle_{\tau, k}=\frac{1}{\tau} \sum_{i \in I_{k}} z_{i}
$$

We then define an accumulated deviation from the mean as

$$
Z_{i, k}=\sum_{j=\ell_{k}}^{i}\left(z_{j}-\langle z\rangle_{\tau, k}\right),
$$

( $\ell_{k}$ labelling the left end of $I_{k}$ ), and from this accumulated deviation we extract the range

$$
R_{\tau, k}=\max _{i \in I_{k}} Z_{i, k}-\min _{i \in I_{k}} Z_{i, k}
$$

while the standard deviation is calculated from the series itself,

$$
S_{\tau, k}=\sqrt{\frac{1}{\tau} \sum_{i \in I_{k}}\left(z_{i}-\langle z\rangle_{\tau, k}\right)^{2}} .
$$

Finally, we calculate the rescaled range $R_{\tau, k} / S_{\tau, k}$, and take its average over all nonoverlapping intervals, obtaining

$$
\rho(\tau) \equiv \frac{1}{n_{\tau}} \sum_{k} \frac{R_{\tau, k}}{S_{\tau, k}},
$$

in which $n_{\tau}=\lfloor L / \tau\rfloor$ is the (integer) number of nonoverlapping intervals of size $\tau$ than can be fit onto a time series of length $L$.

For a purely stochastic curve, with no underlying trends, the rescaled range should satisfy the scaling form

$$
\rho(\tau) \sim \tau^{H},
$$

where $H$ is the Hurst exponent.

2.2. Detrended-Fluctuation Analysis. The detrendedfluctuation analysis (DFA) [9] aims at improving the evaluation of correlations in a time series by eliminating trends in the data. In particular, when a global trend is superimposed on a noisy signal, DFA is expected to provide a more precise estimate of the Hurst exponent than R/S analysis.

The method consists initially in obtaining a new integrated series $Z_{i}$,

$$
Z_{i}=\sum_{j=1}^{i}\left(z_{j}-\langle z\rangle\right)
$$

the average $\langle z\rangle$ being taken over all points,

$$
\langle z\rangle=\frac{1}{L} \sum_{i=1}^{L} z_{i} .
$$

After dividing the series into intervals, the points inside a given interval $I_{k}$ are fitted by a polynomial curve of degree $l$. One usually considers $l=1$ or $l=2$, corresponding to firstand second-order fits. Then, a detrended variation function $\Delta_{i, k}$ is obtained by subtracting from the integrated data the local trend as given by the fit. Explicitly, we define

$$
\Delta_{i, k}=Z_{i}-Z_{i, k}^{f},
$$


where $Z_{i, k}^{f}$ is the value associated with point $i$ according to the fit inside $I_{k}$. Finally, we calculate the root-mean-square fluctuation $F_{\tau, k}$ inside an interval as

$$
F_{\tau, k}=\sqrt{\frac{1}{\tau} \sum_{i \in I_{k}} \Delta_{i, k}^{2}}
$$

and average over all intervals, obtaining

$$
F(\tau)=\frac{1}{n_{\tau}} \sum_{k} F_{\tau, k}
$$

For a true fractal curve, $F(\tau)$ should behave as

$$
F(\tau) \sim \tau^{\alpha}
$$

where $\alpha$ is a scaling exponent. If the trend is correctly identified, one should expect $\alpha$ to be a good approximation to the Hurst exponent $H$ of the underlying correlated noise.

2.3. Box-Counting Analysis. This is a well-known method of estimating the fractal dimension of a point set [7], and it works by counting the minimum number $N(\tau)$ of boxes of linear dimension $\tau$ needed to cover all points in the set. For a real fractal, $N(\tau)$ should follow a power law whose exponent is the box-counting dimension $D_{B}$,

$$
N(\tau) \sim \tau^{-D_{B}} .
$$

For stochastic Gaussian processes, the box-counting and the Hurst exponents are related by

$$
D_{B}=2-H \text {. }
$$

2.4. Minimal-Cover Analysis. This recently introduced method [10] relies on the calculation of the minimal area necessary to cover a given plane curve at a specified scale given by the window size $\tau$.

After dividing the series, we can associate with each interval $I_{k}$ a rectangle of height $H_{k}$, defined as the difference between the maximum and minimum values of the series $z_{i}$ inside the interval,

$$
H_{k}=\max _{i_{0} \leqslant \leq i \leq \leqslant i_{0}+\tau-1} z_{i}-\min _{i_{0} \leqslant \leq i \leq \leqslant i_{0}+\tau-1} z_{i}
$$

in which $i_{0}$ corresponds to the left end of the interval. The minimal area is then given by

$$
A(\tau)=\tau \sum_{k} H_{k}
$$

the summation running over all cells.

Ideally, in the scaling region, $A(\tau)$ should behave as

$$
A(\tau) \sim \tau^{2-D_{\mu}},
$$

where $D_{\mu}$ is the minimal cover dimension, which is equal to 1 when the signal presents no fractality. For genuine fractal curves, it can be shown that, in the limit of infinitely many points, the box-counting and minimal-cover dimensions coincide [10].
2.5. Detrended Cross-Correlation Analysis. This is a recently introduced [11] extension of DFA, based on detrended covariance calculations, and is designed to investigate powerlaw correlations between different simultaneously recorded time series $\left\{x_{i}\right\}$ and $\left\{y_{i}\right\}$.

The first step of the method involves building the integrated time series

$$
X_{j}=\sum_{i=1}^{j} x_{i}, \quad Y_{j}=\sum_{i=1}^{j} y_{i} .
$$

Both series are then divided into $N-(\tau-1)$ overlapping intervals of size $\tau$, and, inside each interval $I_{k}$, local trends $X_{j, k}^{f}$ and $Y_{j, k}^{f}$ are evaluated by least-square linear fits. The detrended cross-correlation $C_{\tau, k}$ is defined as the covariance of the residuals in interval $I_{k}$,

$$
C_{\tau, k}=\frac{1}{\tau} \sum_{j \in I_{k}}\left(X_{j}-X_{j, k}^{f}\right)\left(Y_{j}-Y_{j, k}^{f}\right),
$$

which is then averaged to yield a detrended cross-correlation function

$$
C(\tau)=\frac{1}{N-\tau+1} \sum_{k} C_{\tau, k} .
$$

\section{Pattern-Classification Tools}

Having obtained curves of different fluctuation estimates $Q(\tau)$ as functions of the time interval size $\tau$, we make use of standard pattern-recognition tools in order to group the signals according to relevant classes. The first step towards classification is to build feature vectors from one or more fluctuation analyses of a given signal. In the simplest case, a set of $d$ fixed interval sizes $\left\{\tau_{j}\right\}$ is selected, and the values of the corresponding functions $Q\left(\tau_{j}\right)$ at each $\tau_{j}$, as calculated for the $i$ th signal, define the feature (column) vector $\mathbf{x}_{i}$ of that signal,

$$
\mathbf{x}_{i}=\left(\begin{array}{c}
Q\left(\tau_{1}\right) \\
Q\left(\tau_{2}\right) \\
\vdots \\
Q\left(\tau_{d}\right)
\end{array}\right)
$$

In our studies, unless stated otherwise, we select as interval sizes the nearest integers obtained from powers of $2^{1 / 4}$, starting with $\tau_{1}=4$ and ending with $\tau_{d}$ equal to the length of the shortest series available.

It is also possible to concatenate vectors obtained from more than one fluctuation analysis to obtain feature vectors of larger dimension. This usually leads to better classifiers.

The following subsections discuss different methods designed to group feature vectors into relevant classes. All methods initially select a subset of the available vectors as a training group in order to build the classifier, whose generalizability is then tested with the remaining vectors. This procedure has to be repeated for many distinct choices of training and testing vectors, as a way to evaluate the average efficiency of the classifier. One can then study the resulting confusion matrices, which report the percentage of vectors of a given class assigned to each of the possible classes. 
3.1. Principal-Component Analysis. Given a set of $N$ feature vectors $\left\{\mathbf{x}_{i}\right\}$, principal-component analysis (PCA) is based on the projection of those vectors onto the directions defined by the eigenvectors of the covariance matrix

$$
\mathbf{S}=\frac{1}{N} \sum_{i=1}^{N}\left(\mathbf{x}_{i}-\mathbf{m}\right)\left(\mathbf{x}_{i}-\mathbf{m}\right)^{T}
$$

in which $\mathbf{m}$ is the average vector,

$$
\mathbf{m}=\frac{1}{N} \sum_{i=1}^{N} \mathbf{x}_{i}
$$

and $T$ denotes the vector transpose. If the eigenvalues of $\mathbf{S}$ are arranged in decreasing order, the projections along the first eigenvector, corresponding to the largest eigenvalue, define the first principal component, and account for the largest variation of any linear function of the original variables. In general, the $n$th principal component is defined by the projections of the original vectors along the direction of the $n$th eigenvector. Therefore, the principal components are ordered in terms of the (decreasing) amount of variation of the original data for which they account.

Thus, PCA amounts to a rotation of the coordinate system to a new set of orthogonal axes, yielding a new set of uncorrelated variables, and a reduction on the number of relevant dimensions, if one chooses to ignore principal components whose corresponding eigenvalues lie below a certain limit.

A classifier based on PCA can be built by using the first few principal components to define modified vectors, whose class averages are determined from the vectors in the training group. Then, a testing vector $\mathbf{x}$ is assigned to the class whose average vector lies closer to $\mathbf{x}$ within the transformed space. This is known as the nearest-class-mean rule, and would be optimal if the vectors in different classes followed normal distributions.

3.2. Karhunen-Loéve Transformation. Although very helpful in visualizing the clustering of vectors, PCA ignores any available class information. The Karhunen-Loéve (KL) transformation, in its general form, although similar in spirit to PCA, does take class information into account. The version of the transformation employed here $[8,12]$ relies on the compression of discriminatory information contained in the class means.

The KL transformation consists in first projecting the training vectors along the eigenvectors of the within-class covariance matrix $\boldsymbol{S}_{W}$, defined by

$$
\mathbf{S}_{W}=\frac{1}{N} \sum_{k=1}^{N_{C}} \sum_{i=1}^{N_{k}} y_{i k}\left(\mathbf{x}_{i}-\mathbf{m}_{k}\right)\left(\mathbf{x}_{i}-\mathbf{m}_{k}\right)^{T}
$$

where $N_{C}$ is the number of different classes, $N_{k}$ is the number of vectors in class $k$, and $\mathbf{m}_{k}$ is the average vector of class $k$. The element $y_{i k}$ is equal to one if $\mathbf{x}_{i}$ belongs to class $k$, and zero otherwise. We also rescale the resulting vectors by a diagonal matrix built from the eigenvalues $\lambda_{j}$ of $\boldsymbol{S}_{W}$. In matrix notation, this operation can be written as

$$
\mathbf{X}^{\prime}=\boldsymbol{\Lambda}^{-1 / 2} \mathbf{U}^{T} \mathbf{X}
$$

in which $\mathbf{X}$ is the matrix whose columns are the training vectors $\mathbf{x}_{i}, \Lambda=\operatorname{diag}\left(\lambda_{1}, \lambda_{2}, \ldots\right)$, and $\mathbf{U}$ is the matrix whose columns are the eigenvectors of $\boldsymbol{S}_{W}$. This choice of coordinates makes sure that the transformed within-class covariance matrix corresponds to the unit matrix. Finally, in order to compress the class information, we project the resulting vectors onto the eigenvectors of the between-class covariance matrix $\mathbf{S}_{B}$,

$$
\mathbf{S}_{B}=\sum_{k=1}^{N_{C}} \frac{N_{k}}{N}\left(\mathbf{m}_{k}-\mathbf{m}\right)\left(\mathbf{m}_{k}-\mathbf{m}\right)^{T},
$$

where $\mathbf{m}$ is the overall average vector. The full transformation can be written as

$$
\mathbf{X}^{\prime \prime}=\mathbf{V}^{T} \Lambda^{-1 / 2} \mathbf{U}^{T} \mathbf{X}
$$

$\mathbf{V}$ being the matrix whose columns are the eigenvectors of $\boldsymbol{S}_{B}$ (calculated from $\mathbf{X}^{\prime}$ ).

With $N_{C}$ possible classes, the fully-transformed vectors have at most $N_{C}-1$ relevant components. We then classify a testing vector $\mathbf{x}_{i}$ using the nearest-class-mean rule.

\section{Applications}

4.1. Cast-Iron Microstructure from Ultrasonic Backscattered Signals. An early application of the ideas described in this review aimed at distinguishing microstructure in graphite cast iron through Hurst and detrended-fluctuation analyses of backscattered ultrasonic signals.

As detailed in [2], backscattered ultrasonic signals were captured with a $5 \mathrm{MHz}$ transducer, at a sampling rate of $40 \mathrm{MHz}$, from samples of vermicular, lamellar, and spheroidal graphite cast iron. Double-logarithmic plots of the resulting R/S and DFA calculations, shown in Figure 1, reveal that in all cases two regimes can be identified, reflecting short- and long-time structure of the signals, respectively. From the discussion in Sections 2.1 and 2.2, this implies that one can define two sets of exponents, related to the short- and long-time fractal dimensions of the signals, as estimated from the corresponding values of the Hurst exponent $H$ and the DFA exponent $\alpha$. See (16).

Lamellar cast iron is readily identified as having smaller short- than long-time fractal dimension, contrary to both vermicular and spheroidal cast irons. These latter types, in turn, can be identified on the basis of the relative values of $H$ and $\alpha$ on the different regimes.

As discussed in the following subsections, this fortunate clear distinction on the basis of a very small set of exponents is not possible in more general applications. Nevertheless, a set of relevant features can still be extracted from fluctuation or fractal analyses by using tools from the pattern recognition literature. 


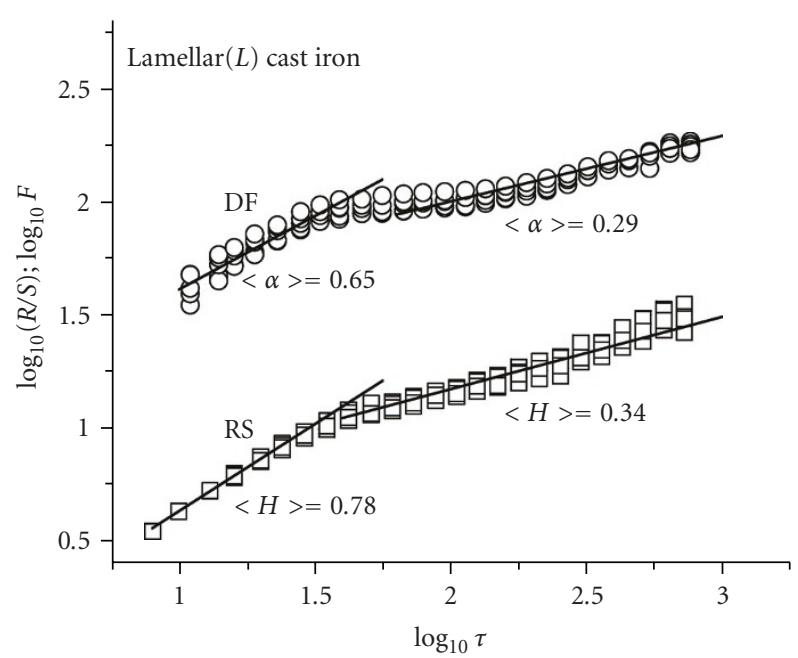

(a)

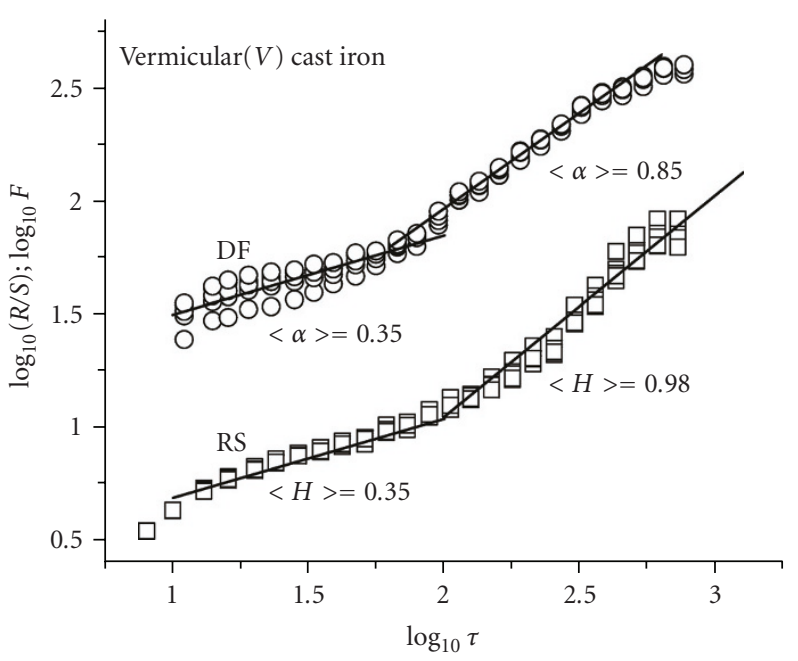

(b)

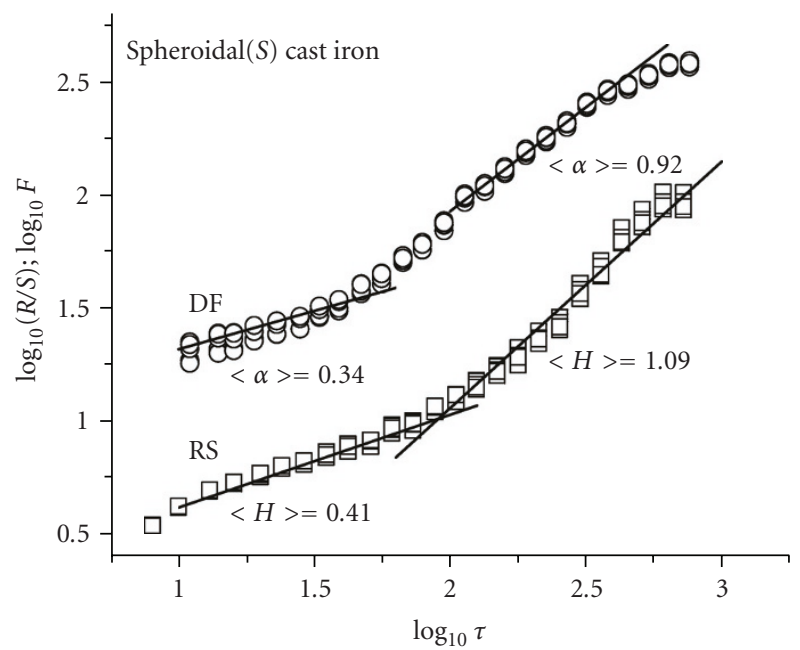

(c)

FIGURE 1: Double-logarithmic plots of the curves obtained from Hurst (R/S) and detrended-fluctuation (DF) analyses of backscattered ultrasonic signals propagating in lamellar (a), vermicular (b), and spheroidal (c) cast iron. The values of $\langle\alpha\rangle$ and $\langle H\rangle$ are obtained by averaging the slopes of all curves in the corresponding intervals, as shown by the solid lines.

4.2. Welding Defects in Metals from TOFD Ultrasonic Inspection. The TOFD (time-of-flight diffraction) technique aims at estimating the size of a discontinuity in a material by measuring the difference in time between ultrasonic signals scattering off the opposite tips of the discontinuity. For welding-joint inspection, the conventional setup consists of one emitter and one receiver transducer, aligned on either side of the weld bead. (Longitudinal rather than transverse waves are used, for a number of reasons, among which is higher propagation speed.)

In the case studied in [13], 240 signals of ultrasound amplitude versus time were captured, with a TOFD setup, from twelve test samples of steel plate AISI 1020, welded by the shielded process. (Details on materials and methods can be found in [14].) The signals used in the study were extracted from sections with no visible defects in the welding, and from sections exhibiting lack of penetration, lack of fusion, and porosities. Each of the four classes was represented by 60 signals, each one containing 512 data points, with 8-bit resolution. Examples of signals from each class are shown in Figure 2.

By combining curves obtained from Hurst, linear detrended-fluctuation, minimal cover, and box-counting analyses into single vectors representing each ultrasonic signal, a very efficient classifier is built using features extracted from a Karhunen-Loéve transformation and the nearestclass-mean rule. The average confusion matrix obtained from 500 sets of 48 testing vectors is shown in Table 1. A maximum error of about $27 \%$ is obtained, corresponding to the misclassification of porosities. A slightly poorer performance is obtained by first building feature vectors from each of the four fluctuation analyses, performing provisional classifications, and then deciding on the final classification by means of a majority vote (with ties randomly resolved). In this 


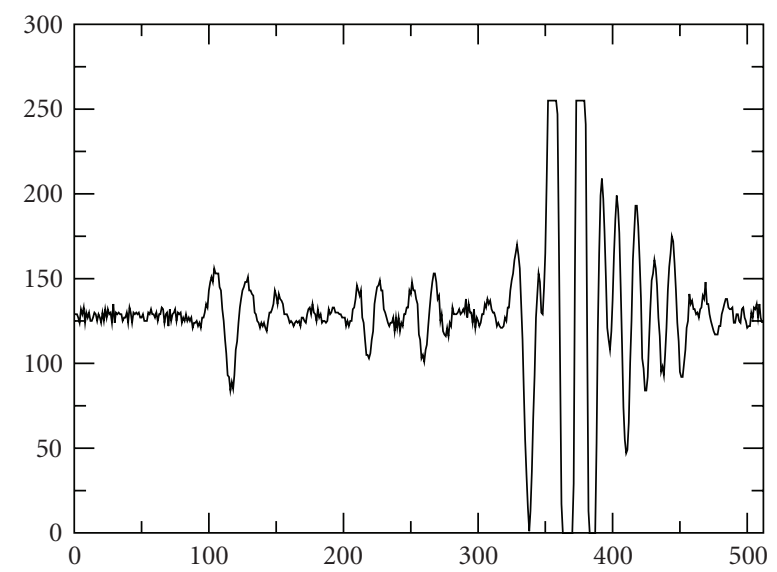

(a)

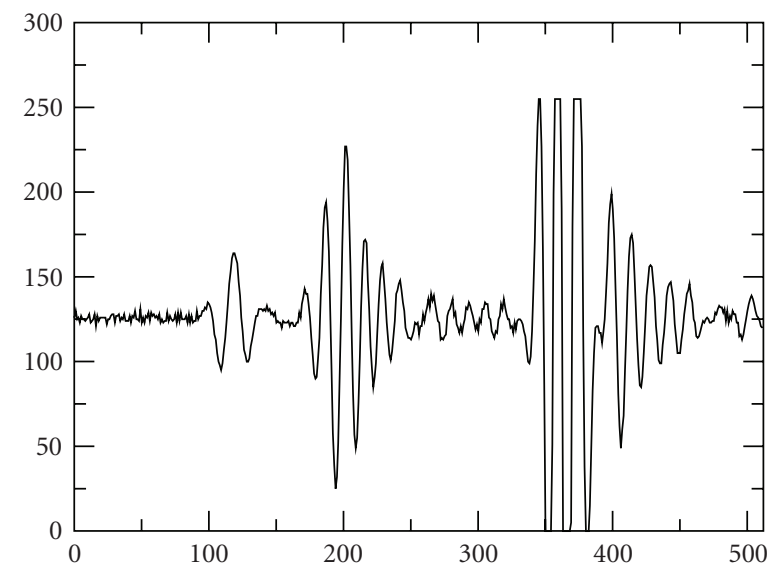

(c)

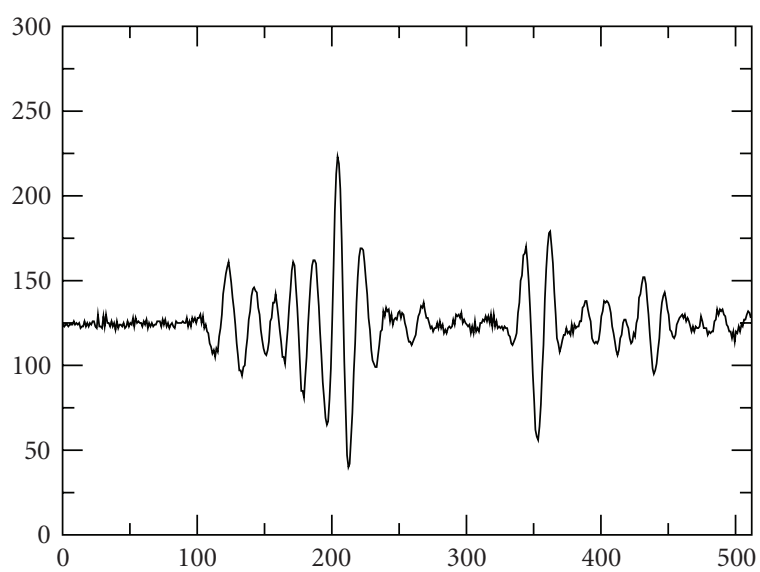

(b)

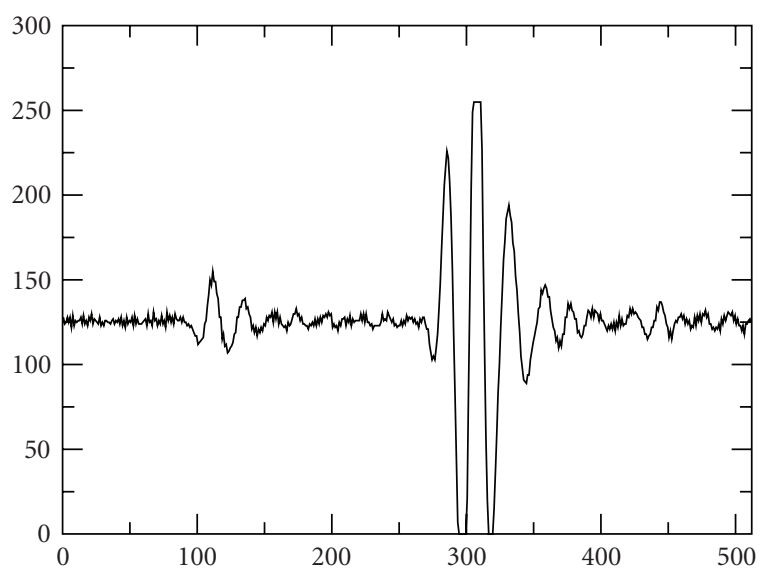

(d)

FIgURE 2: Typical examples of signals obtained from samples with (a) lack-of-fusion defects, (b) lack-of-penetration defects, (c) porosities, and (d) no defects. The horizontal axes correspond to the time direction, in units of the inverse sample rate of the equipment.

TABLE 1: Average percentage confusion matrix for testing vectors built from a combination of fluctuation analyses. The possible classes are lack of fusion (LF), lack of penetration (LP), porosity (PO), and no defects (ND). Figures in parenthesis indicate the standard deviations, calculated over 500 sets. (Notice that in [13] these figures were erroneously reported.) The value in row $i$, column $j$ indicates the percentage of vectors belonging to class $i$ which were associated with class $j$.

\begin{tabular}{lcccc}
\hline & LF & LP & PO & ND \\
\hline LF & $\mathbf{9 1 . 0 7 ( 0 . 3 7 )}$ & $1.69(0.16)$ & $6.88(0.33)$ & $0.35(0.08)$ \\
LP & $2.61(0.37)$ & $\mathbf{8 3 . 9 6 ( 0 . 4 5 )}$ & $12.14(0.41)$ & $1.28(0.14)$ \\
PO & $6.43(0.32)$ & $13.99(0.47)$ & $72.66(0.58)$ & $6.92(0.34)$ \\
ND & $1.01(0.15)$ & $2.55(0.20)$ & $6.92(0.32)$ & $\mathbf{8 9 . 5 1 ( 0 . 4 0 )}$ \\
\hline
\end{tabular}

case, as shown in Table 2, the overall error rate is somewhat increased, although the classification error of samples associated with lack of penetration decreases. In any case, both of these approaches yield considerably better performance than classifiers based on either correlograms or Fourier spectra of the signals, and at a smaller computational cost.
4.3. Gear Faults from Vibration Signals. As detailed in [15], vibration signals were captured by an accelerometer attached to the upper side of a gearbox containing four gears, one of which was sometimes replaced by a gear either containing a severe scratch over 10 consecutive teeth, or missing one tooth.

Several working conditions were studied, consisting of different choices of rotation frequency (from $400 \mathrm{rpm}$ to $1400 \mathrm{rpm}$ ) and to the presence or absence of a fixed external load. For each working condition, 54 signals containing 2048 points were captured (with a sampling rate of $512 \mathrm{~Hz}$ ), 18 signals corresponding to each of the three possible classes of gear (normal, scratched, or toothless). Linear DFA was then performed on the signals, and feature vectors were built from curves corresponding to 13 interval sizes $\tau$ ranging from 4 to 32 . Figure 3 shows representative signals obtained under load, at a rotation frequency of $1400 \mathrm{rpm}$, along with the corresponding DFA curves.

Principal-component analysis was applied to the resulting vectors, and a nearest-class-mean classifier was built from the first three principal components of 36 randomly chosen training vectors. With averages taken over 100 
TABLE 2: The same as in Table 1, but now for a majority vote involving classifications based on each fluctuation analysis separately.

\begin{tabular}{lcccc}
\hline & LF & LP & PO & ND \\
\hline LF & $\mathbf{8 7 . 1 1 ( 0 . 4 0 )}$ & $0.64(0.10)$ & $6.96(0.33)$ & $5.28(0.27)$ \\
LP & $2.04(0.18)$ & $\mathbf{9 0 . 0 6 ( 0 . 4 0 )}$ & $5.88(0.34)$ & $2.01(0.18)$ \\
PO & $7.13(0.34)$ & $19.16(0.52)$ & $\mathbf{6 5 . 1 8}(0.61)$ & $8.53(0.35)$ \\
ND & $2.26(0.19)$ & $1.38(0.17)$ & $7.81(0.34)$ & $\mathbf{8 8 . 5 4 ( 0 . 4 1 )}$ \\
\hline
\end{tabular}

TABLE 3: Average percentage of correctly classified testing signals coming from toothless and normal gears working in the absence of load.

\begin{tabular}{lcccccc}
\hline rpm & 400 & 600 & 800 & 1000 & 1200 & 1400 \\
\hline Toothless & $69.4 \pm 1.9$ & $86.3 \pm 1.5$ & $96.2 \pm 0.7$ & $49.2 \pm 2.9$ & $68.8 \pm 2.1$ & $48.2 \pm 2.5$ \\
Normal & $69.3 \pm 1.8$ & 100 & 100 & $64.1 \pm 2.4$ & $91.5 \pm 1.2$ & $45.1 \pm 2.5$ \\
\hline
\end{tabular}

choices of training and testing vectors, the classifier was always capable of correctly identifying scratched gears, while the classification error of testing vectors corresponding to normal or toothless gears, although unacceptably high for two working conditions in the absence of load, lay below $6 \%$ for most conditions under load. See Tables 3 and 4 . Although a similar classifier based on Fourier spectra yields superior performance, this comes at a much higher computational cost, since feature vectors now have 1024 points [15].

\subsection{Weld-Transfer Mode from Current and Voltage Time} Series. As detailed in [16], voltage and current data were captured during Metal Inert/Active Gas welding of steel workpieces, with a simultaneous high-speed video footage, allowing identification of the instantaneous metal-transfer mode. The sampling rate was $10 \mathrm{kHz}$, and a collection of nine voltage and current time series was built, with three series corresponding to each of three metal-transfer modes (dip, globular, and spray). The typical duration of each series was 4.5 seconds, and examples are shown in Figure 4.

A systematic classification study was performed by first dividing each time series into smaller series containing $L$ points ( $L$ being $512,1024,2048$, or 4096). These smaller series were then processed with Hurst, linear detrended-fluctuation, and detrended-cross-correlation analyses. Figure 5 shows example curves. Selecting $80 \%$ of the obtained feature vectors for training (with averages over 100 random choices of training and testing sets), classifiers were built from voltage or current signals separately processed with Hurst or detrended-fluctuation analyses, as well as from voltage and current signals simultaneously processed with detrended-cross-correlation analysis. A Karhunen-Loéve transformation was finally employed along with the nearest-class-mean rule. In the poorest performance, obtained from signals with $L=512$ points subject to Hurst analysis, the maximum classification error was $27 \%$ for signals corresponding to spray transfer mode, with $100 \%$ correctness achieved for globular transfer mode.

Table 5 shows the average classification error of each classifier, for different series length $L$. The overall performance of classifiers with $L=1024$ and $L=2048$ is better than with the other two lengths. This can be traced to the fact that, as illustrated by Figure 5, distinguishing features (such as average slopes and discontinuities) between curves corresponding to different transfer modes tend to happen at intermediate time scales. For a given length, detrendedcross-correlation analysis of voltage and current signals yields an intermediate classification efficiency as compared to either voltage or current signals analyzed separately. The best classifier is obtained with the Hurst analysis of signals containing $L=2048$ points, yielding a negligible classification error of $0.1 \%$.

In contrast, as shown in the bottom two rows of Table 5, similar classifiers in which feature vectors are defined by the full Fourier spectra of the various signals yield much larger classification errors, and at a much higher computational cost (since the size of feature vectors scales as $L$, whereas for fluctuation analyses it scales as $\log L$ ).

4.5. Stainless Steel Microstructure from Magnetic Measurements. Barkhausen noise is a magnetic phenomenon produced when a variable magnetic field induces magnetic domain wall movements in ferromagnetic materials. These movements are discrete rather than continuous, and are caused by defects in the material microstructure, generating magnetic pulses that can be measured by a coil placed on the material surface.

Magnetic Barkhausen noise (BN) and magnetic flux (MF) measurements were performed on samples of stainlesssteel steam-pressure vessels, as detailed in [17]. These presented coarse ferritic-pearlitic phases (named stage "A") before degradation. Owing to temperature effects, two different microstructures were obtained from pearlite that has partially (stage "BC") or completely (stage "D") transformed to spheroidite. Measurements were performed by using a sinusoidal magnetic wave of frequency $10 \mathrm{~Hz}$, each signal consisting of 40000 points, with a sampling rate of $200 \mathrm{kHz}$. A total of 144 signals were captured, 40 signals corresponding to stage A, 88 to stage $\mathrm{BC}$, and 16 to stage $\mathrm{D}$. Typical signals are shown in Figure 6. Notice that, as regards the magnetic flux, the difference between signals from the various stages seems to lie on the intensity of the peaks and troughs, 


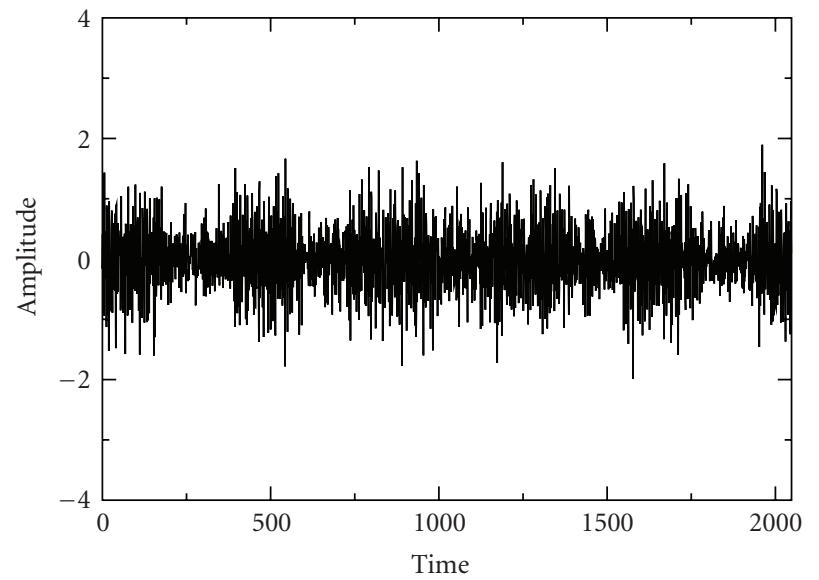

(a) Signal from normal gear

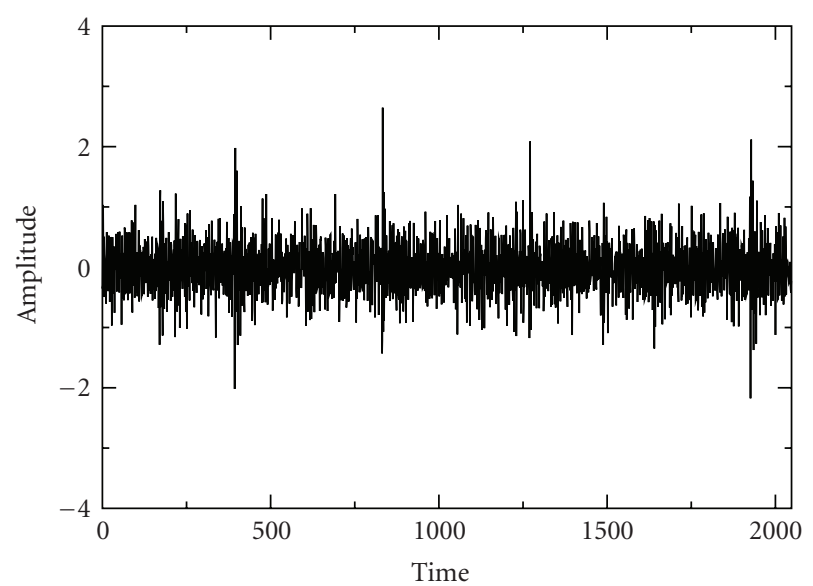

(c) Signal from toothless gear

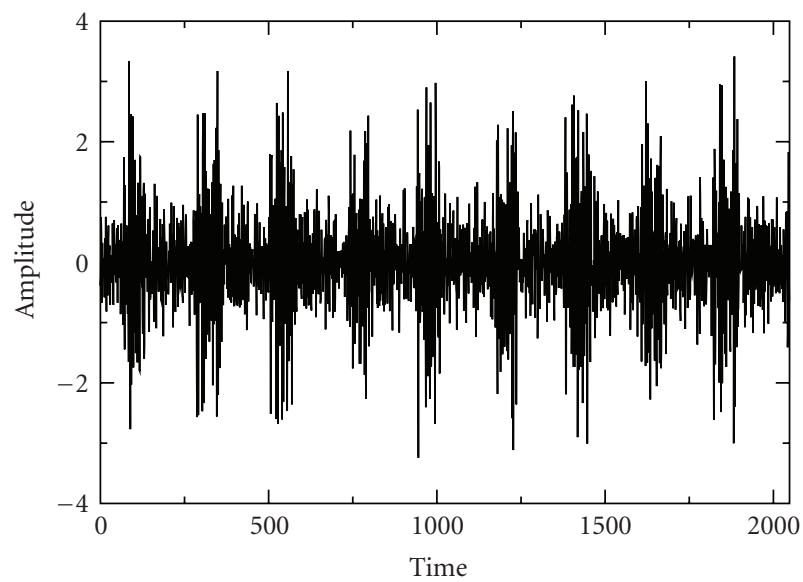

(e) Signal from scratched gear

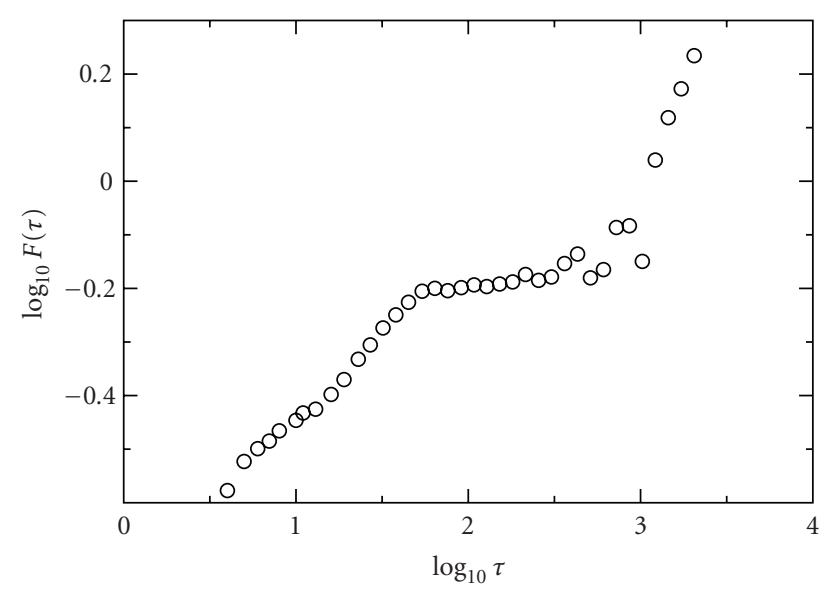

(b) DFA from normal gear

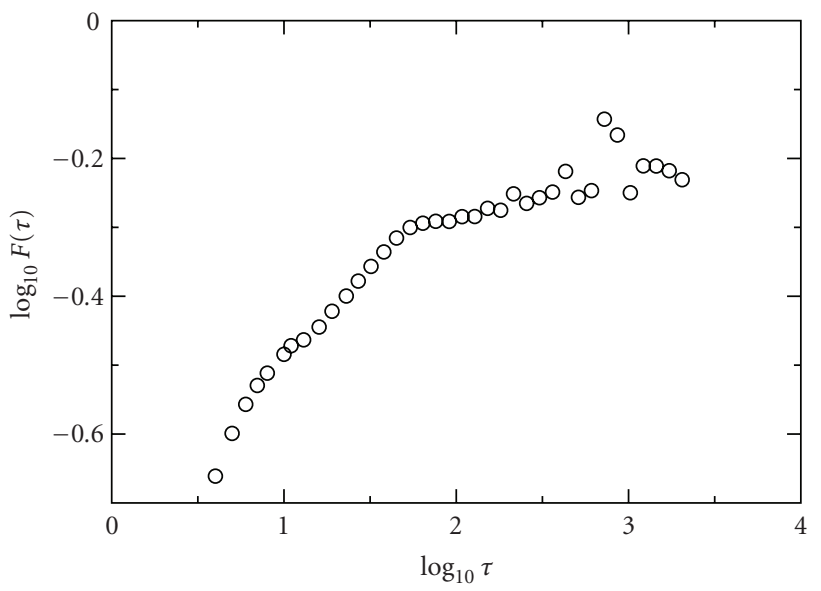

(d) DFA from toothless gear

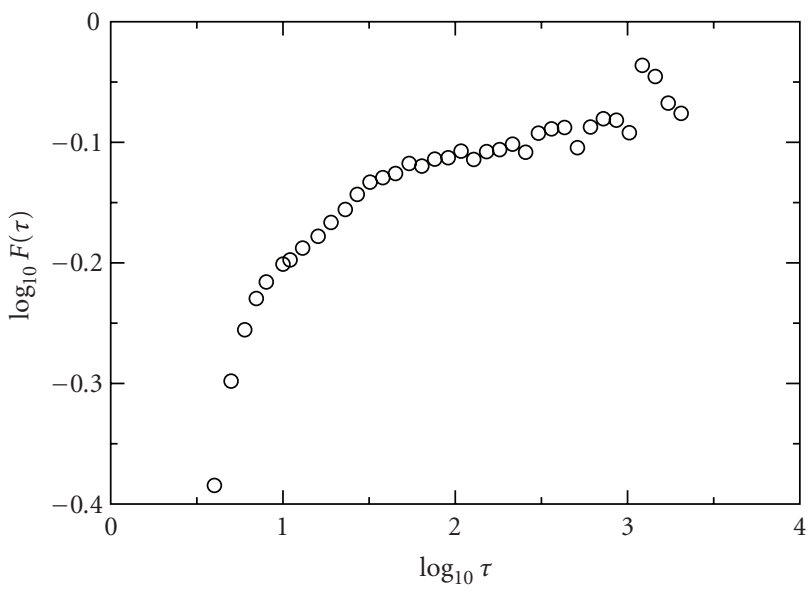

(f) DFA from scratched gear

FIGURE 3: Representative signals and DFA curves obtained from the three types of gear, working under load at a rotation frequency of $1400 \mathrm{rpm}$. In the signal plots, time is measured in units of the inverse sampling rate.

TABLE 4: The same as in Table 3, but now for gears working under load.

\begin{tabular}{lcccccc}
\hline rpm & 400 & 600 & 800 & 1000 & 1200 & 1400 \\
\hline Toothless & 100 & 100 & 100 & 100 & 100 & 100 \\
Normal & $94.8 \pm 0.8$ & $97.5 \pm 0.7$ & $98.5 \pm 0.5$ & $95.6 \pm 0.7$ & $81.3 \pm 1.7$ & 100 \\
\hline
\end{tabular}




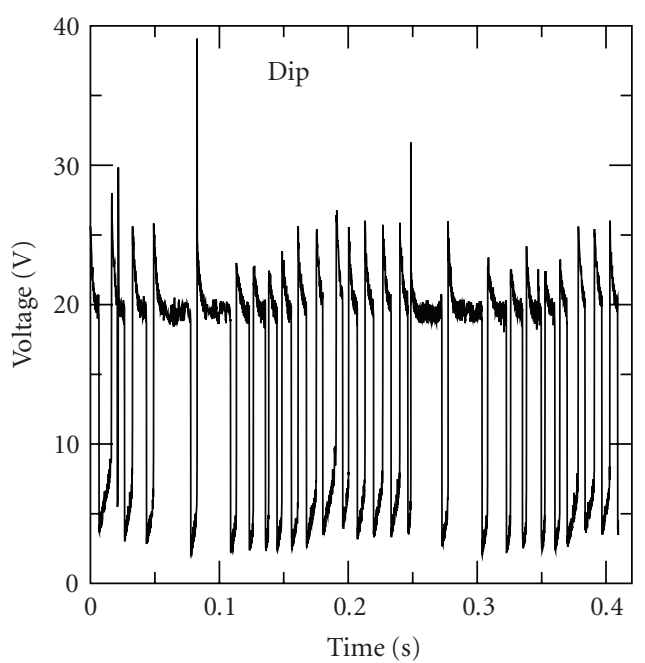

(a)

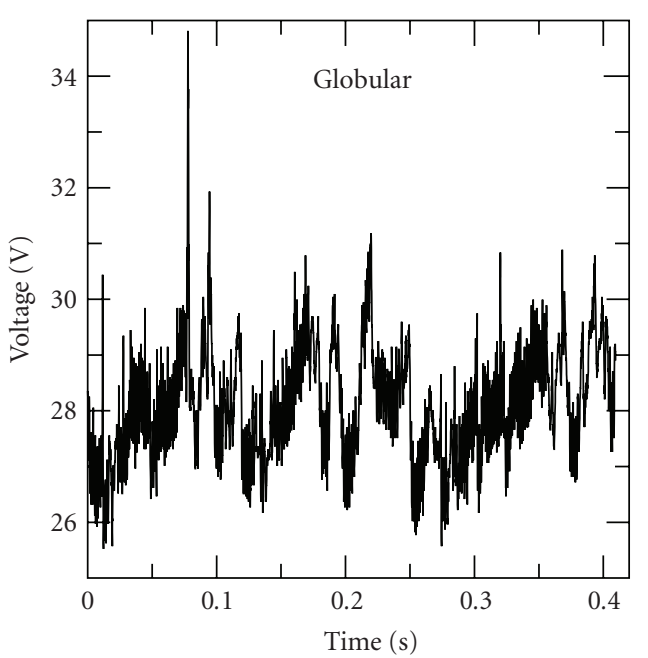

(c)

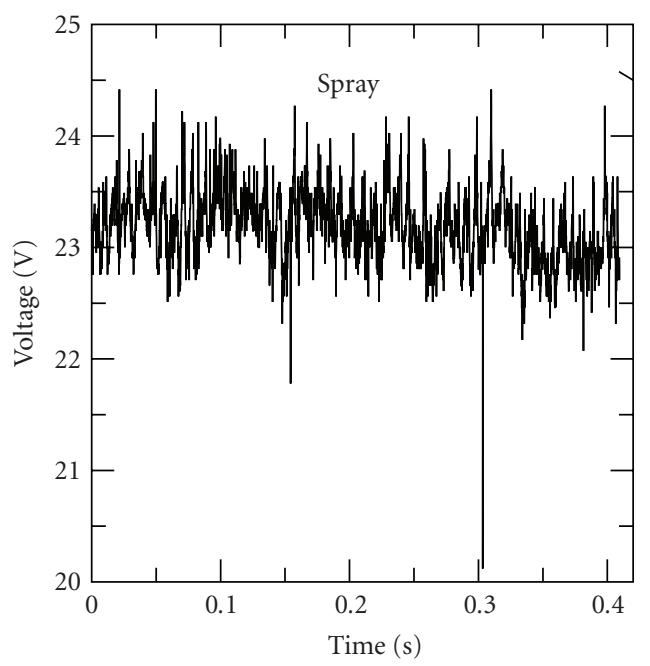

(e)

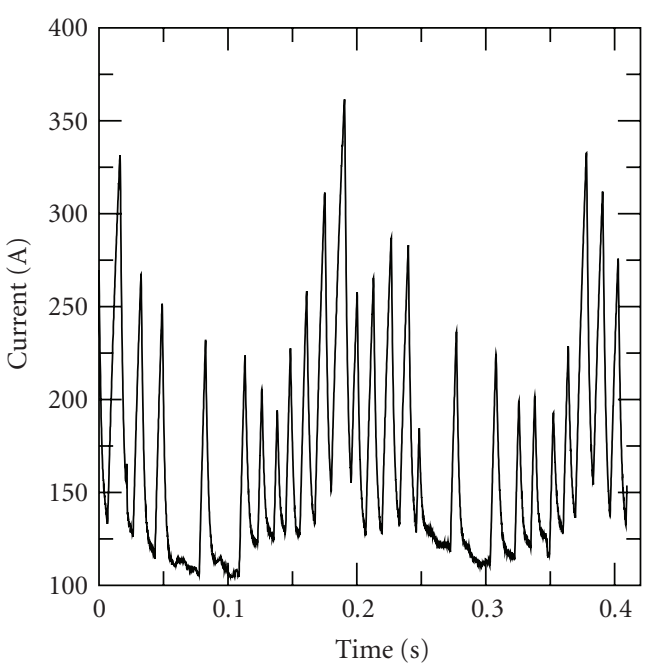

(b)

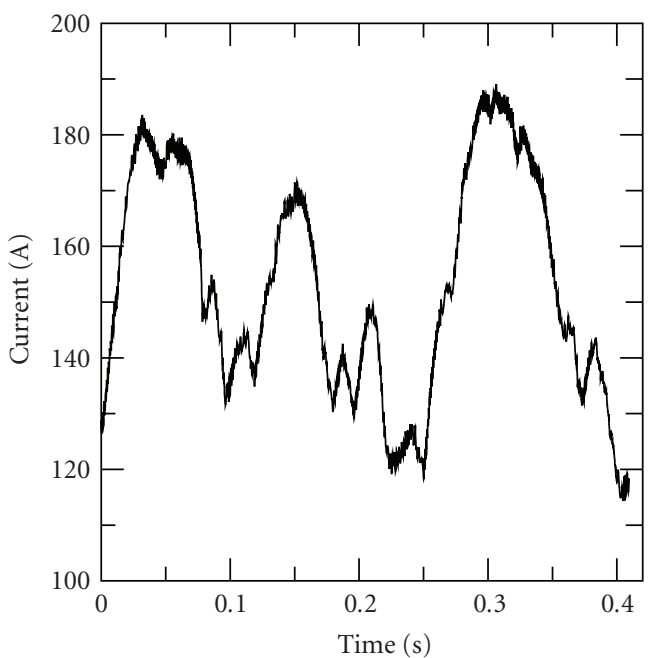

(d)

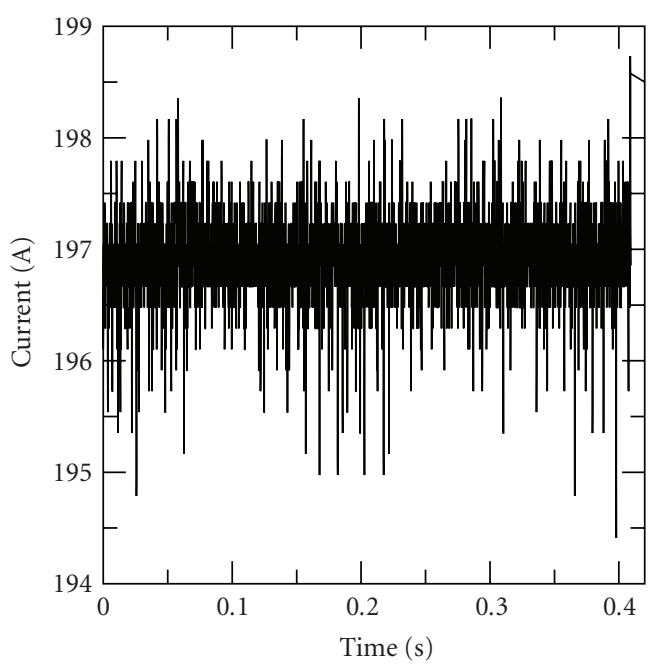

(f)

FIgURE 4: Examples of voltage (left) and current (right) time series obtained during the welding process under dip (top), globular (center), and spray (bottom) metal-transfer modes. 


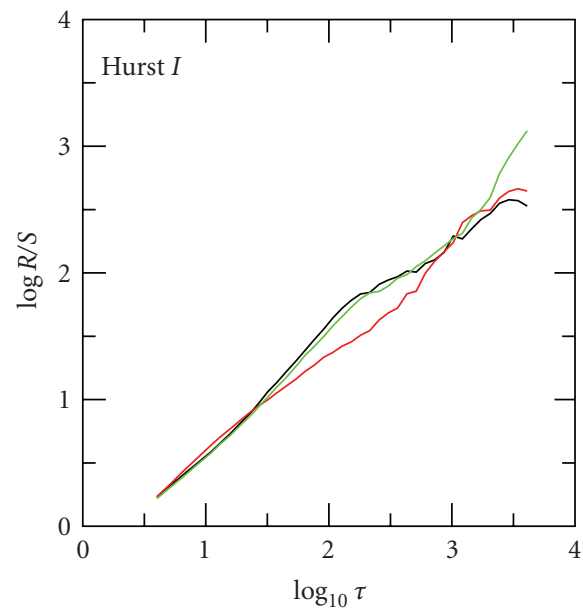

(a)

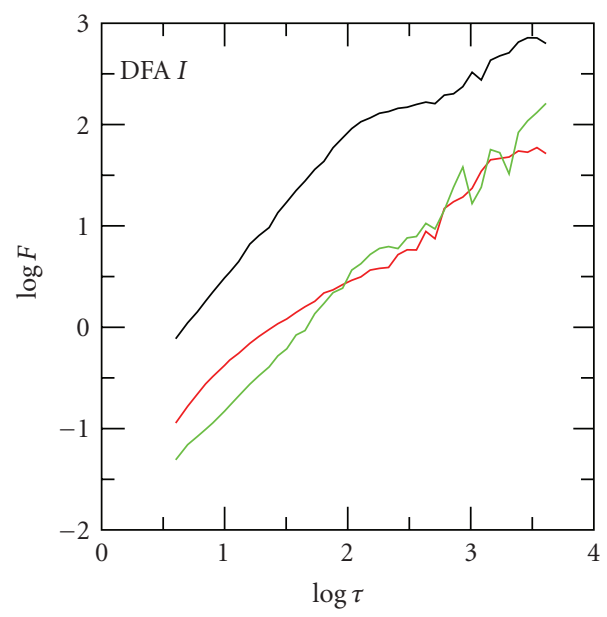

(c)

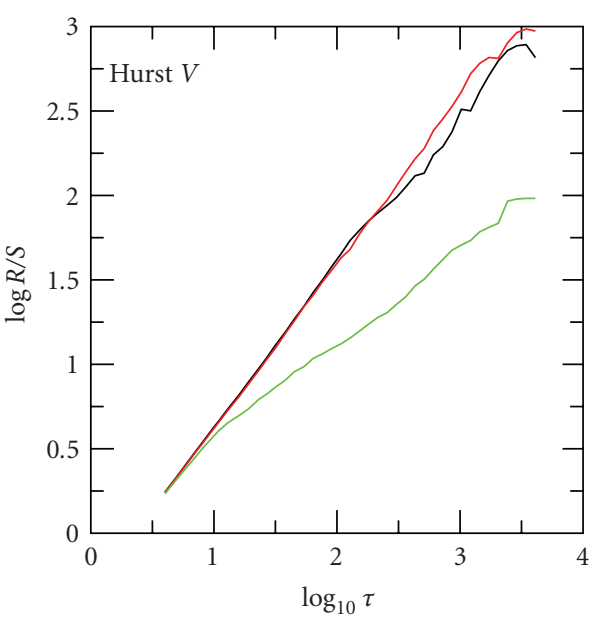

(b)

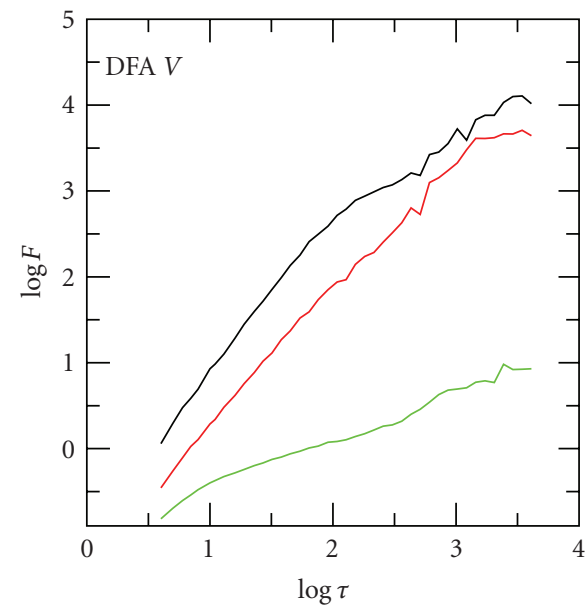

(d)

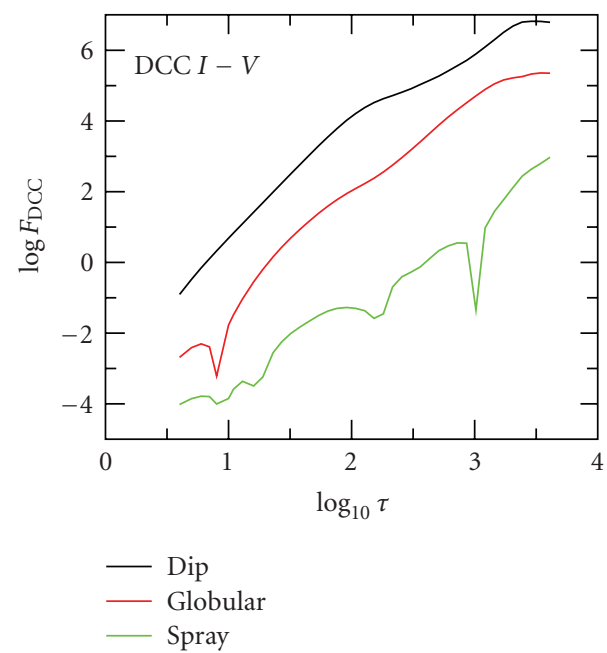

(e)

Figure 5: Examples of curves obtained from Hurst (top), detrended-fluctuation (center), and detrended-cross-correlation (bottom) analyses to current $(I)$ and voltage $(V)$ sample signals obtained under dip (top), globular (center), and spray (bottom) metal-transfer modes. Logarithms are in base 10, and the time window size is measured in tenths of a millisecond. 


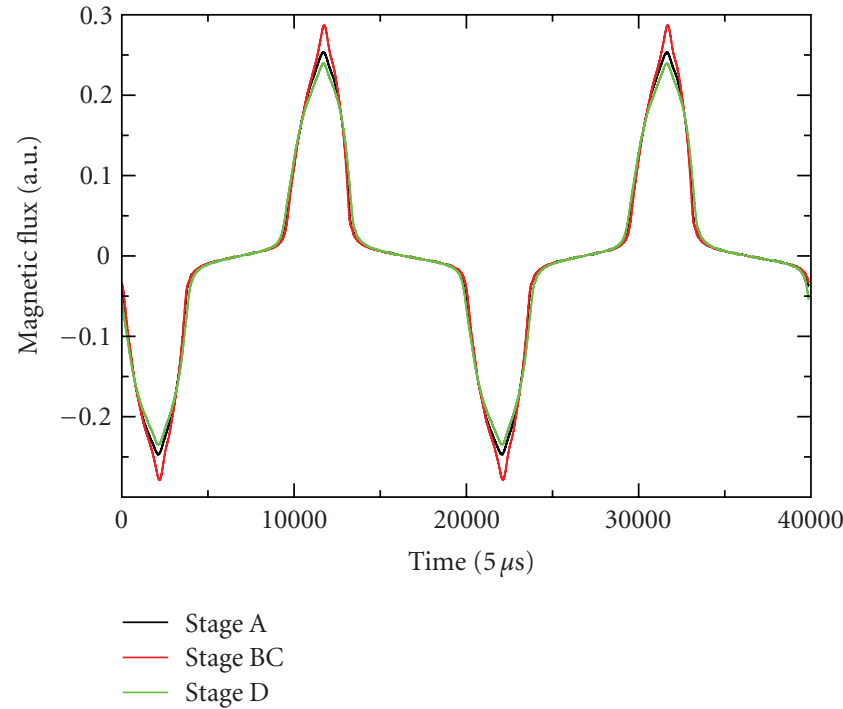

(a)

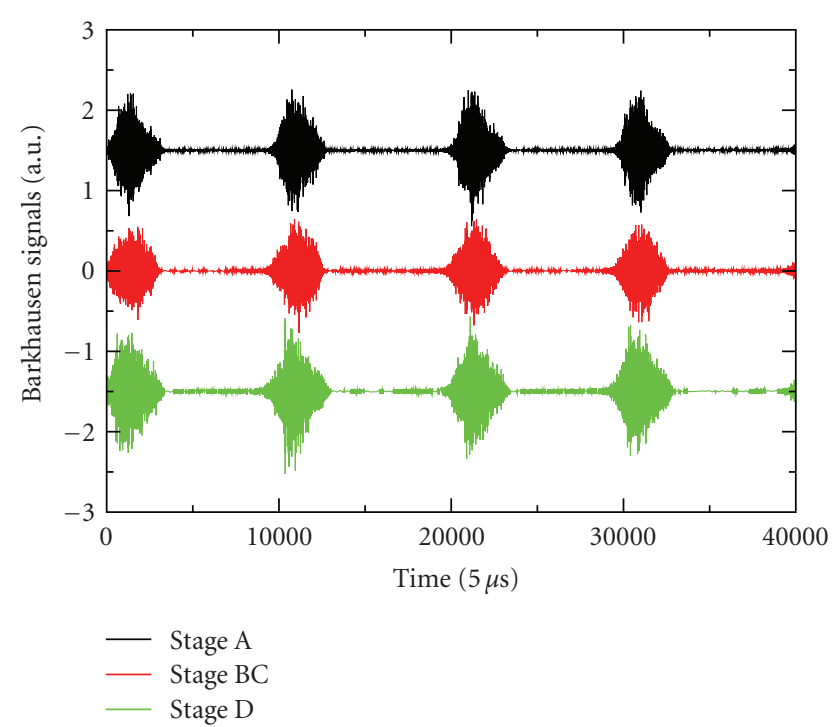

(b)

Figure 6: Typical signals of (a) magnetic flux and (b) Barkhausen noise obtained from stainless-steel samples at different stages of microstructural degradation. Plots in (b) have been vertically shifted for clarity.

TABLE 5: Average percentage classification errors of testing voltage $(V)$ and current $(I)$ signals containing $L$ points, produced by classifiers based on Hurst, detrended-fluctuation (DF), or detrended-cross-correlation (DCC) analyses. Also shown are results for classifiers based on Fourier spectra.

\begin{tabular}{lcccc}
\hline$L$ & 512 & 1024 & 2048 & 4096 \\
\hline DF, $V$ & $3.1 \pm 0.2$ & $2.2 \pm 0.4$ & $3.6 \pm 0.7$ & $5.3 \pm 1.3$ \\
Hurst, $V$ & $6.5 \pm 0.4$ & $3.1 \pm 0.5$ & $0.1 \pm 0.1$ & $0.7 \pm 0.7$ \\
DF, $I$ & $2.1 \pm 0.2$ & $0.6 \pm 0.2$ & $0.5 \pm 0.3$ & $1.6 \pm 1.1$ \\
Hurst, $I$ & $14.5 \pm 0.5$ & $5.4 \pm 0.6$ & $4.0 \pm 0.9$ & $2.7 \pm 1.3$ \\
DCC, $V+I$ & $3.2 \pm 0.3$ & $1.5 \pm 0.3$ & $2.4 \pm 0.7$ & $7.7 \pm 1.3$ \\
\hline Fourier, $V$ & $23.6 \pm 0.9$ & $21.8 \pm 0.8$ & $18.7 \pm 1.2$ & $36.7 \pm 2.3$ \\
Fourier, $I$ & $22.7 \pm 2.5$ & $27.5 \pm 1.9$ & $8.7 \pm 0.9$ & $14.5 \pm 1.9$ \\
\hline
\end{tabular}

TABLE 6: Average percentage of correctly classified testing signals coming from stainless-steel samples in different degradation stages. Classifiers employed detrended-fluctuation (DFA), Hurst (RS), or Fourier spectral (FS) analyses on either Barkhausen noise (BN) or magnetic flux (MF).

\begin{tabular}{lccccc}
\hline & DFA/BN & RS/BN & DFA/MF & RS/MF & FS/MF \\
\hline Stage A & $54.8 \pm 1.9$ & $34.2 \pm 1.6$ & $83.0 \pm 1.3$ & $90.5 \pm 1.0$ & $67.8 \pm 1.7$ \\
Stage BC & $57.6 \pm 1.2$ & $49.5 \pm 1.5$ & $87.2 \pm 0.8$ & $92.5 \pm 0.6$ & $77.0 \pm 1.1$ \\
Stage D & $68.4 \pm 2.9$ & $31.0 \pm 2.7$ & $96.4 \pm 1.5$ & $98.0 \pm 1.4$ & $78.6 \pm 2.9$ \\
\hline
\end{tabular}

although there is also a fine structure in the curves which is not visible at the scale of the figure.

Results from classifiers based on detrended-fluctuation and Hurst analyses, with a KL transformation as the final step, are shown in Table 6, for both BN and MF signals, with averages over 100 sets of training and testing vectors. Also shown for comparison are results from classifiers based on Fourier spectral analysis (making use of magnetic-flux signals with 512 points extracted from the original signals by selecting every 78th point, in order to build feature vectors with a manageable number of dimensions). The performance of classifiers based on Barkhausen noise is much inferior to that of classifiers based on magnetic flux signals, which is now discussed. 
The best performance is obtained by the Hurst classifier, with maximum error of about $10 \%$, followed by the DFA classifier, with a maximum error around $17 \%$. Somewhat surprisingly, in view of the long-time regularity of the magnetic flux signals evident in Figure 6, the Fourierspectral classifier shows the worst performance, with an average classification error of $25 \%$.

\section{Conclusions}

We have reviewed and supplemented recent work on application of fluctuation analysis as a pattern-classification tool in nondestructive materials inspection. This approach has been shown to lead to very efficient classifiers, with a performance comparable, and usually quite superior, to more traditional approaches based, for instance, on Fourier transforms. The present approach also requires less computational effort to achieve a given efficiency, which would be an important issue when building automated inspection systems for field work.

An extension of the present approach to defect recognition from radiographic or ultrasonic images can be achieved based on generalizations of the fluctuation analyses to measure surface roughness $[18,19]$. Given any two-dimensional image, a corresponding surface can be built by a color-toheight conversion procedure, and mathematical analyses can then be performed.

\section{Acknowledgments}

The authors acknowledge financial support from the Brazilian agencies FUNCAP, CNPq, CAPES, FINEP (CT-Petro), and Petrobras (Brazilian oil company).

\section{References}

[1] P. Barat, "Fractal characterization of ultrasonic signals from polycrystalline materials," Chaos, Solitons \& Fractals, vol. 9, no. 11, pp. 1827-1834, 1998.

[2] J. M. O. Matos, E. P. de Moura, S. E. Krüger, and J. M. A. Rebello, "Rescaled range analysis and detrended fluctuation analysis study of cast irons ultrasonic backscattered signals," Chaos, Solitons \& Fractals, vol. 19, no. 1, pp. 55-60, 2004.

[3] F. E. Silva, L. L. Gonçalves, D. B. B. Fereira, and J. M. A. Rebello, "Characterization of failure mechanism in composite materials through fractal analysis of acoustic emission signals," Chaos, Solitons \& Fractals, vol. 26, no. 2, pp. 481-494, 2005.

[4] H. E. Hurst, "Long-term storage capacity of reservoirs," Transactions of the American Society of Civil Engineers, vol. 116, pp. 770-799, 1951.

[5] J. Feder, Fractals, Plenum Press, New York, NY, USA, 1988.

[6] B. B. Mandelbrot and J. W. van Ness, "Fractional brownian motion, fractional noises and applications," SIAM Review, vol. 10, pp. 422-437, 1968.

[7] P. S. Addison, Fractals and Chaos, IOP, London, UK, 1997.

[8] A. R. Webb, Statistical Pattern Recognition, John Wiley \& Sons, West Sussex, UK, 2nd edition, 2002.

[9] C.-K. Peng, S. V. Buldyrev, S. Havlin, M. Simons, H. E. Stanley, and A. L. Goldberger, "Mosaic organization of DNA nucleotides," Physical Review E, vol. 49, no. 2, pp. 1685-1689, 1994.
[10] M. M. Dubovikov, N. V. Starchenko, and M. S. Dubovikov, "Dimension of the minimal cover and fractal analysis of time series," Physica A, vol. 339, no. 3-4, pp. 591-608, 2004.

[11] B. Podobnik and H. E. Stanley, "Detrended cross-correlation analysis: a new method for analyzing two nonstationary time series," Physical Review Letters, vol. 100, no. 8, Article ID 084102, 4 pages, 2008.

[12] J. Kittler and P. C. Young, "A new approach to feature selection based on the Karhunen-Loeve expansion," Pattern Recognition, vol. 5, no. 4, pp. 335-352, 1973.

[13] A. P. Vieira, E. P. de Moura, L. L. Gonçalves, and J. M. A. Rebello, "Characterization of welding defects by fractal analysis of ultrasonic signals," Chaos, Solitons \& Fractals, vol. 38, no. 3, pp. 748-754, 2008.

[14] E. P. de Moura, M. H. S. Siqueira, R. R. da Silva, J. M. A. Rebello, and L. P. Calôba, "Welding defect pattern recognition in TOFD signals Part 1. Linear classifiers," Insight, vol. 47, no. 12, pp. 777-782, 2005.

[15] E. P. de Moura, A. P. Vieira, M. A. S. Irmão, and A. A. Silva, "Applications of detrended-fluctuation analysis to gearbox fault diagnosis," Mechanical Systems and Signal Processing, vol. 23, no. 3, pp. 682-689, 2009.

[16] A. P. Vieira, H. H. M. Vasconcelos, L. L. Gonçalves, and H. C. de Miranda, "Fractal analysis of metal transfer in mig/mag welding," in Review of Progress in Quantitative Nondestructive Evaluation, vol. 1096 of AIP Conference Proceedings, pp. 564$571,2009$.

[17] L. R. Padovese, F. E. da Silva, E. P. de Moura, and L. L. Gonçalves, "Characterization of microstructural changes in coarse ferritic-pearlitic stainless steel through the statistical fluctuation and fractal analyses of barkhausen noise," in Review of Progress in Quantitative Nondestructive Evaluation, vol. 1211 of AIP Conference Proceedings, pp. 1293-1300, 2010.

[18] J. A. Tesser, R. T. Lopes, A. P. Vieira, L. L. Gonçalves, and J. M. A. Rebello, "Fractal analysis of weld defect patterns obtained from radiographic tests," in Review of Progress in Quantitative Nondestructive Evaluation, vol. 894 of AIP Conference Proceedings, pp. 539-545, 2007.

[19] G.-F. Gu and W.-X. Zhou, "Detrended fluctuation analysis for fractals and multifractals in higher dimensions," Physical Review E, vol. 74, no. 6, Article ID 061104, 2006. 